\title{
Flex Projector-Interactive Software for Designing World Map Projections
}

\section{Bernhard Jenny Institute of Cartography ETH Zurich, Switzerland jenny@karto.baug.ethz.ch}

Tom Patterson US National Park Service Harpers Ferry, WV, USA tom_patterson@nps.gov

Lorenz Hurni Institute of Cartography ETH Zurich, Switzerland hurni@karto.baug.ethz.ch

INTRODUCTION

"In an era when nearly all aspects of mapmaking are customizable by the user, map projection design has been a bastion of specialization."
Flex Projector is a free, open-source, and cross-platform software application that allows cartographers to interactively design custom projections for small-scale world maps. It specializes in cylindrical, and pseudocylindrical projections, as well as polyconical projections with curved parallels. Giving meridians non-uniform spacing is an option for all classes of projections. The interface of Flex Projector enables cartographers to shape the projection graticule, and provides visual and numerical feedback to judge its distortion properties. The intended users of Flex Projector are those without specialized mathematical expertise, including practicing mapmakers and cartography students. The pages that follow discuss why the authors developed Flex Projector, give an overview of its features, and introduce two new map projections created by the authors with this new software: the A4 and the Natural Earth projection.

Flex Projector is available at www.flexprojector.com.

$\mathrm{D}$ espite the central importance of projections to mapmaking, prior to the release of Flex Projector few cartographers have ever created a map projection. Explanations for this lack of involvement include the ready availability of existing map projections; the time and tedium associated with designing projections, with no guarantee of success; and, the general lack of mathematical expertise needed to devise projections. It is an opaque undertaking to all but a few. Not that these barriers have prevented cartographers from informally experimenting with new projection designs. In the pre-digital era, pencils, graph paper, French curves, and optical devices were the tools of choice for such tinkering. Today, programs such as Adobe Photoshop and Illustrator offer innumerable graphical tools for changing the appearance of a projection with just the click of a mouse. What cartographer in an uninhibited moment has not thought about adjusting the width-to-height proportions of a map so that it would fit better in a graphical layout, or perhaps applying a transformation filter to portray the world with a unique new shape? It is completely natural that mapmakers should want control over the look of world map projections beyond what is possible by adjusting the parameters of existing map projections. In an era when nearly all aspects of mapmaking are customizable by the user, map projection design has been a bastion of specialization.

Taking a cue from the way cartographers work, Flex Projector offers a suite of graphical tools and interactive feedback for the design of custom world map projections. Guiding the software design was the idea that shape and form are the primary determinants for selecting a projection-

Initial submission, December 4, 2007; revised submission, February 29, 2008; final acceptance, March 4, 2008 
an acknowledgement that maps are inherently graphical. Flex Projector, however, is more than just a glorified graphical application for reshaping how the world looks. It alters the internal geometry of existing projections to create new projections, provides the user with detailed information about the angular, areal, and scale distortion properties, imports and exports data in a variety of graphics and GIS formats, and saves new projections as text files that others can reproduce. It is a mapping application.

\section{Lessons from the Robinson Projection}

Flex Projector uses a graphical approach to map projection design similar to that used by Arthur H. Robinson for devising the famous projection that shares his name. In 1961, Robinson was commissioned by Rand McNally to design a world map projection that, among other criteria, was uninterrupted, had limited distortion, and was pleasing to the eye of general viewers (Robinson, 1974). He came up with a very simple idea: instead of devising a mathematical formula that relates longitude and latitude intersections on the sphere to X/Y coordinates on the map, he developed two sets of tabular parameters by trial and error. The first table described the length of parallels for every five degrees of increasing latitude (the horizontal arrows in Figure 1). The second table of parameters defined the distance of each parallel from the equator, also in steps of five degrees of increasing latitude (the vertical arrows in Figure 1). Interpolation determined the coordinates of points for intervals finer than five degrees.

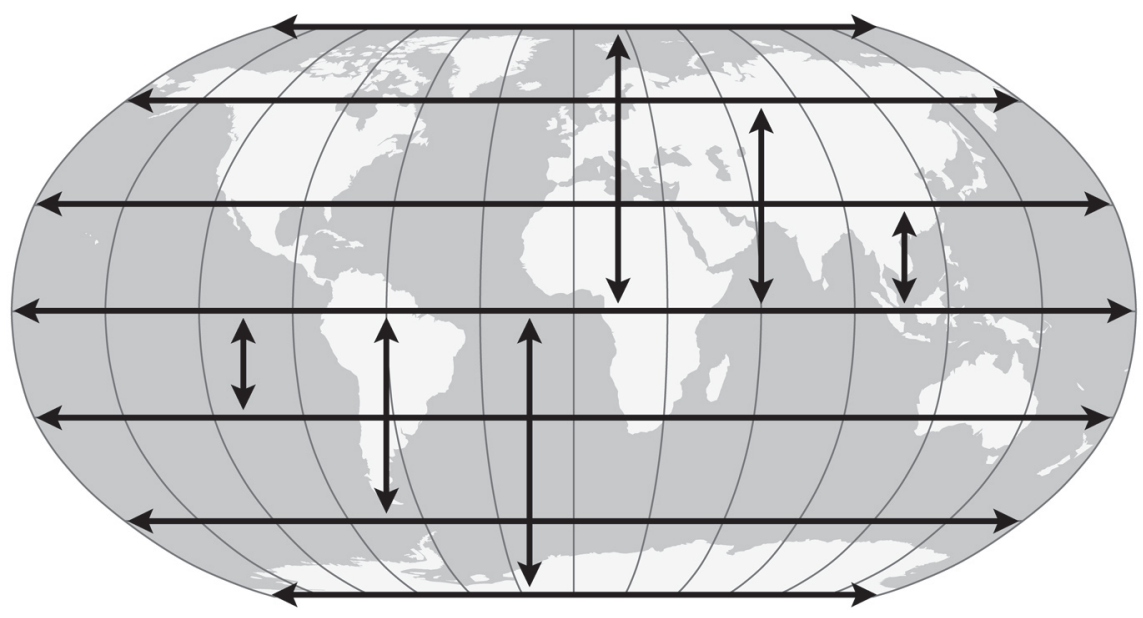

Figure 1. Two sets of tabular parameters define the Robinson projection shown here with horizontal arrows (length of parallels) and vertical arrows (distance of parallels from equator). Note: not all parameters appear in the illustration.

Robinson used an iterative process to create his pseudocylindrical projection, evaluating the appearance and relative relationships of landmasses in a succession of drafts. He started by estimating values for the length and spacing of parallels and then plotted the positions of continents on the resulting graticule. When the look of the projection was less than satisfactory, as was typically the case early on, Robinson made compensating adjustments and drafted a new projection. He repeated this process, a sort of graphic successive approximation, until it became obvious that further adjustments would produce no improvement, at least to the eyes of the author (Robinson, 1974, p. 151-152). The Robinson projection was well received by cartographers and widely used, including by the National Geographic Society (Garver, 1988).
“... instead of devising a mathematical formula that relates longitude and latitude intersections on the sphere to $X / Y$ coordinates on the map, Robinson developed two sets of tabular parameters by trial and error." 
NEW PROJECTIONS NEEDED

"The principal goal of Flex Projector is to give cartographers and the mathematical layperson a means to design new map projections that are pleasing to look at, functional, and minimize shape and area distortion."
Arthur Robinson's task would have been greatly simplified had he employed Flex Projector. The graphical user interface of Flex Projector allows the user to alter the length of parallels and their distance from the equator, just as Robinson did. The results immediately appear on screen with a graticule and sample coastline dataset. Flex Projector extends Robinson's methodology in two major ways. (1) Robinson's projection uses straight parallels. With Flex Projector, bending parallels to concave or convex curves is possible, in a manner similar to the arced parallels seen on the popular Winkel Tripel projection. (2) Robinson's projection distributed meridians with even spacing along the equator. This resulted in a true pseudocylindrical projection, where meridians are equidistant on all parallels (Snyder, 1993, p. 189). Flex Projector provides the option to distribute meridians with uneven spacing.

Popular map projections, which give shape to our mental image of the world, fall in and out of vogue over time. Take for example National Geographic Society (NGS), which used the Van der Grinten I as its world map projection from 1922 to 1988, a notably long run. In 1988, the NGS switched to the Robinson projection, originally called the orthophanic projection, meaning "pleasing to the eye". The Robinson projection is still popular today mostly because of the balanced appearance of major landmasses. It has a classic shape that looks the way a world map should look to the eyes of many readers. The Robinson era at National Geographic, however, came to an end in 1998 when the staff chose the Winkel Tripel projection as its replacement, primarily because its compact form fit better on a two-page atlas spread. Other map publishers have followed suit and the Winkel Tripel has risen from relative obscurity to become common today. Readers of National Geographic will no doubt see a switch to another world map projection in the future.

Considering that hundreds of map projections already exist, is there really a need for an application like Flex Projector? To answer this question one only has to peruse the world maps in popular atlases. Chances are good that you will find only a half-dozen or so map projections in common use, including the Eckert IV, Goode Homolosine, Miller Cylindrical, Mercator, Mollweide, Robinson, and Winkel Tripel projection. This scarcity-amidst-plenty paradox is in part due to the staid preferences of map publishers who are unwilling to risk sales by exposing readers to unfamiliar world map projections. Educational publishers in the US prefer to use only one world map projection in a text for consistency and to avoid confusing students (Bosacki, 2007). Other factors in the lack of diversity are the many published projections designed exclusively for large and medium-scale maps, not small-scale world maps; projections created for purely mathematical reasons and never intended for everyday mapmaking; and projections that are whimsical. For example, the Apple projection, which depicts the world in the shape of an apple with a bite taken out of it (Strebe, 1999), will probably never appear in the National Geographic Atlas of the World. Personal taste is also a major selection criterion; any given cartographer may or may not like an otherwise appropriate projection favored by others. For all of the reasons above, the number of acceptable projections for making world maps for general audiences is small.

The principal goal of Flex Projector is to give cartographers and the mathematical layperson a means to design new map projections that are pleasing to look at, functional, and minimize shape and area distortion. By expanding the pool of people who can design projections, our hope is for a proliferation of new projections tailored to meet the specific needs 
of cartographers. And from this might emerge the next blockbuster world map projection.

A freeware application based on Java 1.5, Flex Projector is cross-platform compatible on Linux, Mac OS X, and Windows. The authors of this article developed the core of the application, including the graphical interface, the algorithms for adjusting the projection, and the code for loading, visualizing and exporting geographical data. The source of projections (other than the Flex Projection created by the user) is Jerry Huxtable's Java port of the widely used PROJ.4 library (Huxtable 2007, Evenden 2005). The user licence allows others to inspect the code, and add their own extensions. Cubic spline interpolation algorithms govern the shape of projections created in Flex Projector; however, the graphic interface shields the average user from this underlying technology.

When designing the interface of Flex Projector, it was the authors' hope that users would have little need for the manual. Upon opening Flex Projector for the first time, the user sees a graphic user interface comprised of three components (Figure 2). The panel in the upper left is a world map in the familiar Robinson projection. To the right of the map is the Flex Projector panel with sliders that control the shape of the projection, and which beckon the user to experiment. Moving any of the sliders results in an immediate change to the Robinson projection, which then ceases to be a Robinson projection and starts on its way to becoming an entirely new projection. Below the map is the Distortion table, which reports in realtime the amount of distortion contained in the modified "Flex" projection, including comparisons to common world map projections.
FLEX PROJECTOR 1.0OVERVIEW

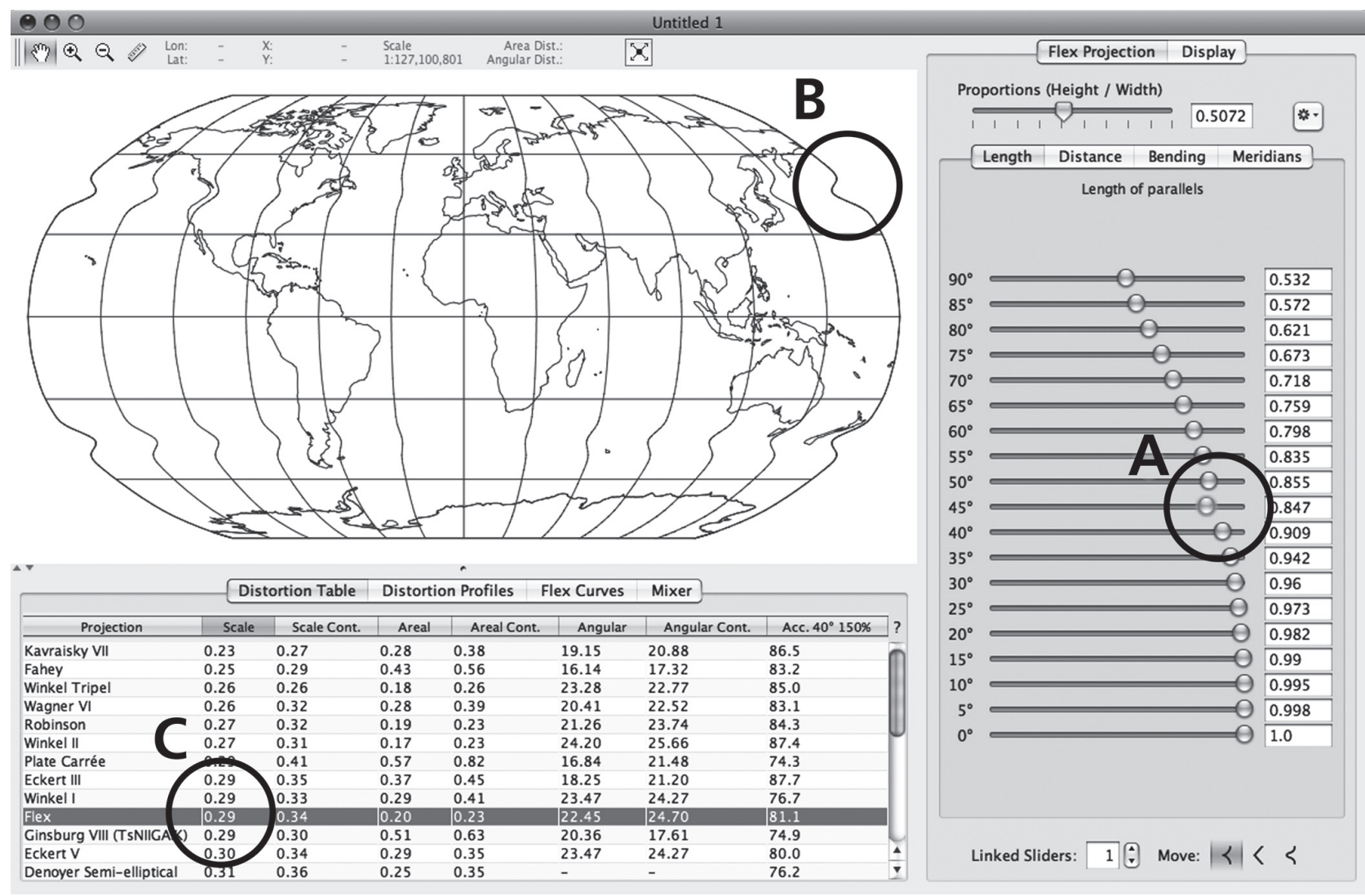

Figure 2. Action-Reaction: Moving a slider (A) in Flex Projector, in this case to the left, results in commensurate changes to the map $(B)$ and in the distortion table (C). 


\section{Flex Projector Panel}

The heart of Flex Projector, the sliders in this panel relate to the upper right (northeast) quadrant of the displayed map projection. Because projections created in Flex Projector have bi-lateral symmetry, the software automatically mirrors the information in the upper right quadrant to calculate the shape of the rest of the world. The four tabs at the top of the panel access sliders for adjusting:

- Length of parallels (see Figure 2 above)

- Distance of parallels from the equator

- Convex or concave bending of parallels

- Distribution of meridians

The Linked Sliders option at the bottom of the panel allows the movement of multiple sliders simultaneously. Increasing the number of linked sliders generally produces projections with smoother, more uniform shapes. The Move Sliders option works in conjunction with Linked Sliders to constrain the movement of sliders to peaked, linear, or bell-curve shapes. An additional slider on the top of the panel, the Proportions slider, offers a quick method to alter the height-to-width ratio of a projection.

With the basic controls described above a user can adjust a projection to an almost infinite variety of shapes, including adjusting the position of the central meridian to any longitude. However, it is not possible to design every type of projection. The current version of Flex Projector is limited to

". . a user can adjust a projection to an almost infinite variety of shapes ..." map projections that show the entire world, have an equatorial aspect (the latitude of origin is always the equator), that are symmetrical relative to the central meridian and the equator, and uninterrupted. (A later version of the application may address some of these limitations). All projections designed in Flex Projector use a spherical earth model. A more complicated ellipsoidal model would not significantly enhance the geometry of small-scale world maps of the type that Flex Projector was conceived to create.

Because mistakes are invariably made while working on a new projection, Flex Projector gives the user unlimited undos to go back to a previous state. If a projection is completely beyond hope, the Options/Reset to Projection button at the top right allows the user to reload a fresh Robinson projection, or one of dozens of other projections.

\section{Display Panel}

The Display panel contains displayable options that aid in the construction of a projection. By clicking the Show Second Projection button, the user can choose a second map projection that appears as a ghosted template behind the current Flex projection. The second projection serves as a visual reference for gauging the design of the Flex projection. The user can change the color of both the Flex and Second projection in the Preferences drop menu.

Turning on Tissot's Indicatrices, Isolines of Areal Distortion, and Isolines of Maximum Angular Distortion shows where distortion occurs in a projection (Figure 3). Also available are controls for setting the graticule density and choosing a central meridian other than the Greenwich Meridian, which is the default.

\section{Distortion Table}

Flex Projector offers various numerical indices for assessing projection distortion (see the bottom-left table in Figure 2): (1) the weighted mean error 


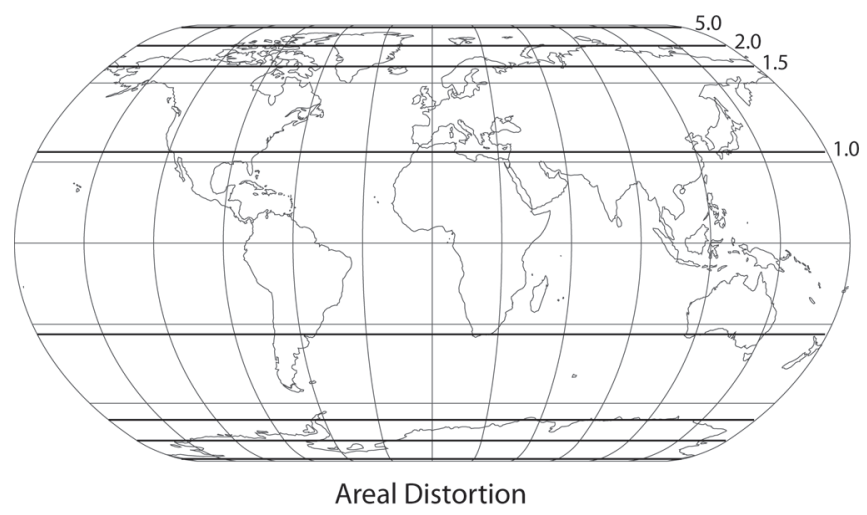

Figure 3: Isolines of areal (left) and angular (right) distortion for the Natural Earth projection.

for overall scale distortion, (2) the weighted mean error for areal distortion, and, (3) the mean angular deformation index. These indices compute distortion for the entire globe and for terrestrial areas only (Canters, 2002). The Acceptance index is an additional numerical measure that summarizes overall projection distortion (Capek, 2001). As the user modifies a Flex projection, the Distortion Table automatically updates every change and ranks the projection compared to other well-known world projections from best (top) to worst (bottom).

Associated with the Distortion table are tabs that display Distortion Profiles and Flex Curves. The information in these graphs alerts the user about otherwise unseen distortion irregularities in a projection.

\section{Data}

Flex Projector reads and projects vector, raster, and elevation data from common GIS and raster formats. The Flex Projector website provides public domain vector, shaded relief, and Natural Earth II data to begin making publication-quality maps. Detailed vector data layers import directly into Flex Projector via the File drop menu. The following data formats can be imported: ESRI Shape for vector data, ESRI ASCII GRID for gridded raster elevation and thematic data, and a variety of image formats, such as JPEG, TIFF, PNG, and BMP. Flex Projector can export to these formats: DXF, ESRI Shape, Adobe Illustrator, JPEG, PDF, PNG, SVG, TIFF, and Ungenerate.

When reprojecting raster data, users have the choice of using nearest neighbor or bi-cubic interpolation. The software assumes that any raster images with a 2:1 aspect ratio are in the Plate Carrée projection and automatically georeferences them. Flex Projector can work with images large enough for wall maps. For example, making the Natural Earth projection (described in a later section) involved reprojecting an image measuring 16,200 x 8,100 pixels in size. Reprojected vector data saved in Adobe Illustrator (AI) format, include a bounding box indicating the maximum area extent. These boxes allow the user to register raster art visually to vector maps in graphical applications.

Map designers can share projections created in Flex Projector by saving the projection parameters as text files that others using Flex Projector can read and use. Projections created in Flex Projector are currently not transferable to other map projection applications.

The steps below outline a sample workflow in Flex Projector that leads to a customized projection we call the "A4" projection (Figure 4), which has similarities to the Winkel Tripel projection. Characteristics of the A4
"The software assumes that any raster images with a 2:1 aspect ratio are in the Plate Carrée projection and automatically georeferences them."

HOW TO DESIGN A NEW MAP PROJECTION 
"Employing an iterative process, the user would assess the shape of the graticule and appearance of major landmasses after each adjustment."

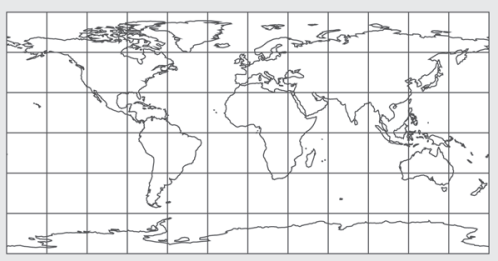

1. Plate Carrée

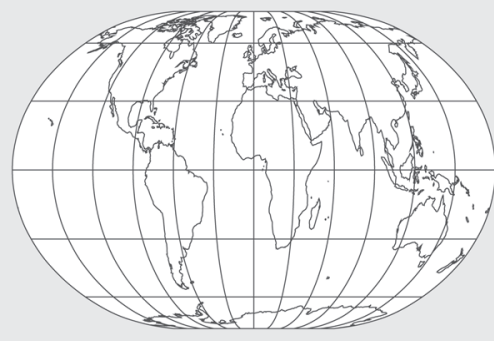

4. Distance of Parallels

projection include a compact form factor, arcing parallels, a straight pole line, and meridians that are regularly spaced along the equator. Designing the A4 projection involved six steps:

1. The procedure started with the Plate Carrée projection opened via the Reset dialog.

2. Increasing the height-to-width proportion from 0.50 to 0.655 made the map fit better on an A4 sheet in landscape format with extra space in the margins.

3. Adjusting the length of parallels (with the Linked Sliders option selected) curved the meridians and the overall shape of the map. Using a repetitive trial-and-error method assured that arcs were smooth and had the desired curvature.

4. Decreasing the distance of parallels from the equator selectively at high latitudes compressed the polar areas, moderating the areal distortion found there.

5. Bending of the parallels reduced the north-south elongation of Africa and South America. The pole lines received no bending.

6. Applying a scale factor of 0.7785 minimized the total areal distortion of the graticule, reducing the apparent scale of the map.

Creating a new projection like the A4 projection described by the simple steps above would, in reality, require frequent use of the undo-redo functionality of Flex Projector to evaluate variations. Employing an iterative process, the user would assess the shape of the graticule and appearance of major landmasses after each adjustment. Distortion information obtained from isolines, indices of distortion, and Tissot's indicatrices would also guide the design decisions.

In a real-world workflow, the recommended way to design a new projection is usually not to start with the Plate Carrée projection, but with a predefined projection closer to that of the desired final. For example, when making the A4 projection, starting with the Winkel Tripel projection would simplify steps 1-4 as described above, avoiding unnecessary major adjustments to the shape of the graticule.

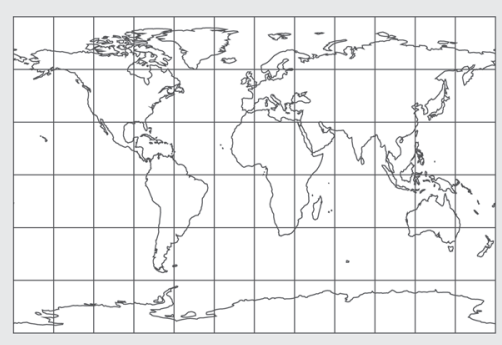

2. Proportions

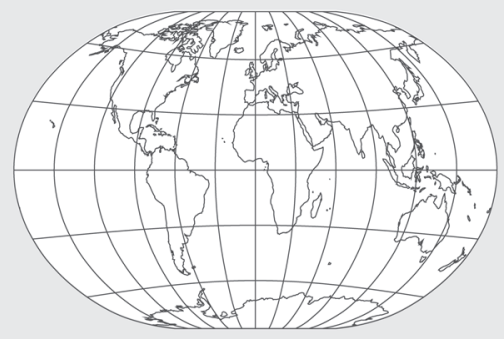

5. Bending of Parallels

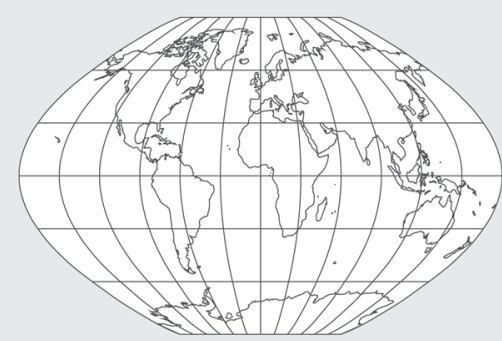

3. Length of Parallels

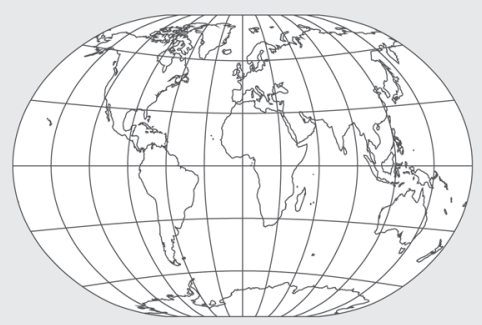

6. Scale Factor

Figure 4. Designing the A4 projection. 
An illustration of the A4 projection and its parameters can be found in Appendix A.

Students and teachers are among the intended users of Flex Projector. With its hands-on interface and interactive feedback, Flex Projector is fun to use and entices students to explore how to make map projections. A mathematical background is not required. For teachers, the software provides a unique environment for devising creative assignments. For example: ask students to design a world map projection for a hypothetical publishing company, similar to what Arthur Robinson did for Rand McNally. When designing a new projection, students would have no choice but to think critically about projection characteristics as part of the creative process. And requiring students to name their creation after themselves, as is the convention with naming projections, would motivate them to do a better job.

Other advantages for education include:

- Learning the importance of defining design objectives before beginning work on a new projection.

- By giving students real-time feedback about angular, areal, and scale distortion, Flex Projector reinforces the idea that every projection design involves making significant compromises.

- Students can compare the projections they make against published projections. Besting the distortion rating of a famous map projection is a worthwhile and achievable objective.

- Universal access: Flex Projector is free, will run on most computers, and uses common data formats.

- Advanced students with computer programming experience can modify the source code to extend the capability of Flex Projector.

That Flex Projector is useful for production cartography should be abundantly clear to those who have read this far. In this section, we discuss the making of a new projection customized to portray the Natural Earth II dataset, from which the projection takes its name (Figure 5). Natural Earth II is a raster map dataset of the planet in the Plate Carrée projection that features natural environment colors, terrestrial shaded relief, and sea floor shaded relief with depth tints (Patterson, 2007).

The impetus for creating the Natural Earth projection was dissatisfaction with existing world map projections for displaying physical data. World physical maps typically employ two classes of projections: cylindrical and pseudocylindrical. Cylindrical projections are widely used for maps with sea floor relief, a preference that is perhaps a throwback to the traditional use of the Mercator projection for ocean navigation. Using the Mercator projection for a reference map, however, is less than ideal because of the extreme areal exaggeration in high latitudes. For example, the World Ocean Floor map published by National Geographic in 1975 uses the Mercator projection and omits areas beyond 75 degrees of latitude, to keep the map to a reasonable size. When making a map with raster digital data the polar problem is even worse because of poor data quality found in these areas, which degrades even further with enlargement. Even the more moderate Miller Cylindrical projection with less polar distortion than the Mercator suffers from this problem. For example, reprojecting raster data from the Plate Carée to the Miller Cylindrical projection stretches the north-south axis of Greenland by nearly 200 percent, damaging image quality in the process. For this reason, and because the world is not rectangular in shape, we removed cylindrical map projections from consideration.
EDUCATIONAL USERS

NATURAL EARTH PROJECTION

"Using the Mercator projection
for a reference map, however,
is less than ideal because of the
extreme areal exaggeration in
high latitudes."

"When designing a new projection, students would have no choice but to think critically about projection characteristics as part of the creative process."

NATURALEARTH PROJECTION 


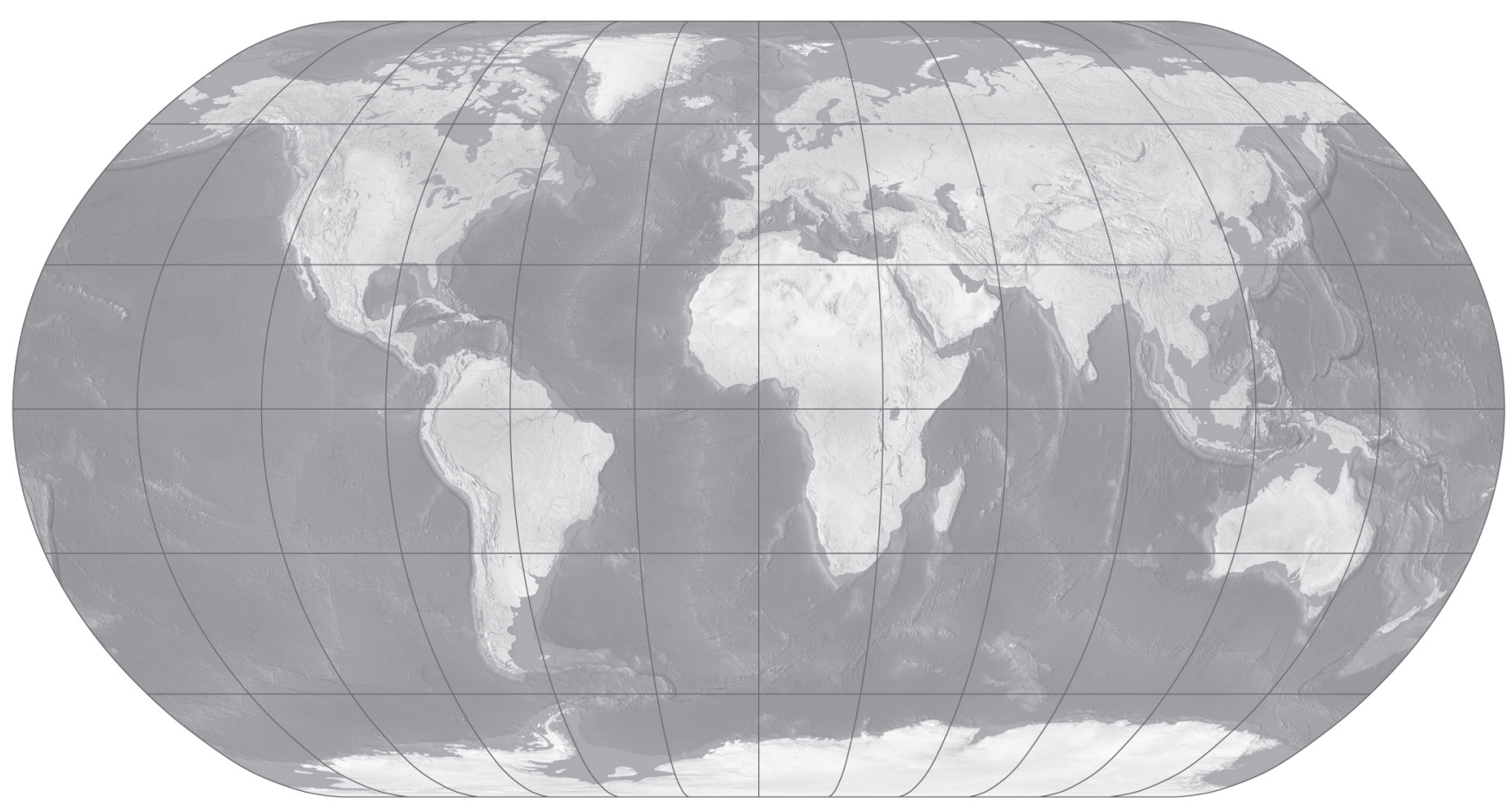

Figure 5. The Natural Earth projection applied to the Natural Earth II dataset. (see page 68 for color version)

Pseudocylindrical projections are better suited for presenting raster data because their arcing meridians converge toward the poles, compressing the size of these areas and tightening image quality. By selectively diminishing the distance of parallels from the equator in high latitudes even more polar compression is possible. An added benefit to curved meridians is a more rounded shape that hints that the projection is a $2 \mathrm{D}$ representation of a 3D sphere. In selecting a pseudocylindrical projection to display the Natural Earth II dataset the following requirements were sought:

- No graticule: creating a projection that could "stand alone" without the supporting framework of a graticule was important. Because pseudocylindrical projections have straight parallels, readers can judge the relative latitude of areas without the presence of a graticule. However, some popular pseudocylindrical projections, for example, the Eckert IV and Mollweide, have ovoid shapes that look too soft and capsule-like without a graticule. The projection needed to have a strong shape.

- Wall map: the Winkel Tripel (not a pseudocylindrical projection because of its curved parallels) and other projections with compact forms apply to situations where space is limited, such as the printed page. By contrast, wall maps are largely free of horizontal space constraints and can afford to portray the world with greater breadth and detail.

- Conventional appearance: a projection with pleasing lines and minimal distortion that would not detract from the Natural Earth II data presented on it was a high priority. As an example of what was not desired, the Sinusoidal projection with its sharply pointed poles and top-like shape would attract unwanted attention to itself. The ideal projection needed to be both functional and rather familiar in appearance. 
The Kavraiskiy VII and Robinson projections—-both of which are compromise projections that are neither conformal nor equal area but are rated well for overall distortion-came closest to fulfilling the above requirements. However, each projection had at least one undesirable characteristic. The Kavraiskiy VII, with its 0.5774 height-to-width proportions, depicts tropical and mid latitude areas with minimal distortion, but exaggerates the size of high latitude areas-Antarctica is enormous. The Robinson projection, with its 0.5072 height-to-width proportions, suffers from the opposite problem: it is slightly too wide and its sides bulge outwards. When centered on the Greenwich Meridian, this results in too much angular distortion in Alaska, Kamchatka, and New Zealand (and the adjacent ocean floor) near the map edges.

Seeking the best characteristics of each, the Natural Earth projection is an amalgam of the Kavraiskiy VII and Robinson projections, plus additional enhancements (Figure 6). Making the Natural Earth projection in Flex Projector started with the Robinson projection. In the first step, the height-to-width proportion was increased from 0.5072 to 0.52 , to give it slightly more height. The Kavraiskiy VII was then loaded as a second projection in the background and given the same width as the Robinson projection. Using the Kavraiskiy VII as a template, the parallels on the Robinson projection were each increased in length to four decimal points of precision, to match the bounding meridians of the Kavraiskiy VII. The projection then took on a completely new form, similar to that of a truncated Kavraiskiy VII projection. The final procedure for creating the Natural Earth projection was decreasing the length of the pole lines by a small amount and giving the corners (where the pole lines and bounding meridians meet) a rounded appearance. This involved trial and error experimentation and hours of contemplative staring at draft projections before deciding on the final (See Appendix B for Natural Earth projection parameters).

\author{
"Seeking the best \\ characteristics of each, the \\ Natural Earth projection is an \\ amalgam of the Kavraiskiy VII \\ and Robinson projections ..."
}

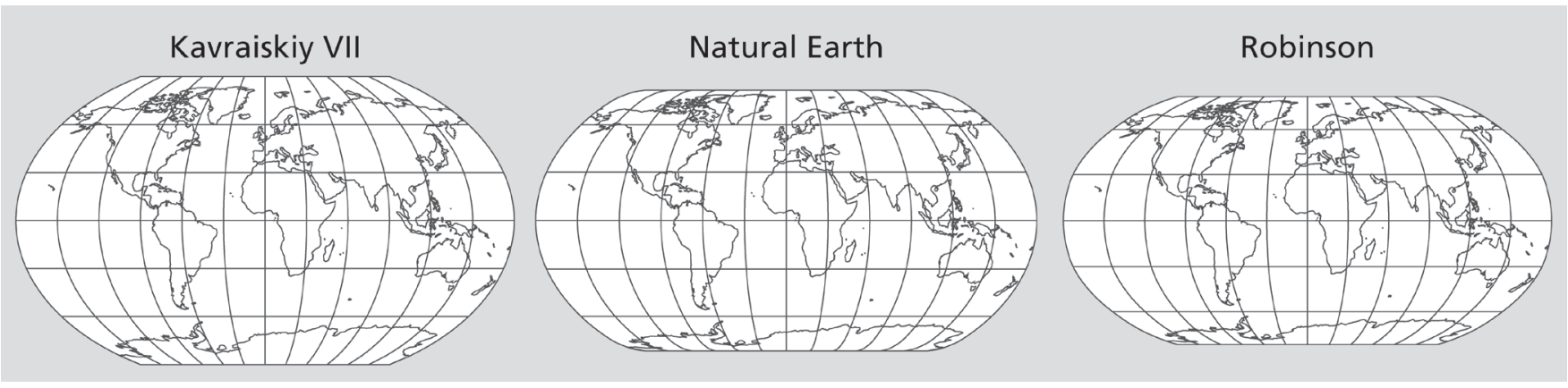

Figure 6. The Natural Earth projection combines characteristics of the Kavraiskiy VII and Robinson projections.

Designing the Natural Earth projection with rounded corners served five purposes:

1) They suggest that the projection represents a spherical Earth.

2) Rounding corners and the related action of lessening the length of pole lines reduced the size of polar areas, thereby making Antarctica appear smaller.

3) At the top and bottom of the projection, meridians converge inward toward implied poles, suggesting that the poles are in fact points instead of straight lines.

4) Aesthetics: from iPod music players, to Jaguar automobiles to the Mona Lisa, curves convey classic elegance. 
"It is the authors' hope that Flex Projector will democratize the creation of world map projections..."

CONCLUSION
5) The tightly rounded corners of the Natural Earth projection are unique among commonly used pseudocylindrical projections, helping to differentiate it.

Because the Natural Earth projection derives from two projections with low overall distortion, its distortion values fall somewhere between those of the Kavraiskiy VII and Robinson. Distortion values for the Natural Earth projection could be slightly better. Graphical considerations, rather than slavish attention to improving distortion, drove the design decisions. The final result was a projection with cleaner lines and whose distortion was still well within acceptable limits (See Appendix C for distortion tables of the Natural Earth projection).

As a compromise projection, the Natural Earth projection is not equal area and in fact exaggerates the size of high latitude areas. Despite our egalitarian desire to show all areas on a map at their true relative sizes, on physical maps exaggerating the size of high latitudes serves a useful purpose. Most land on Earth lies at high latitudes in the northern hemisphere and these areas also have highly complex coasts. Greenland and India, for example, are landmasses of roughly comparable shape and area, one at high latitudes and the other low. The fjorded Greenland coast is 44,000 kilometers in length, while the smooth coast of India measures only 7,000 kilometers. (Taking into account that the northern boundary of India does not include coast, unlike Greenland, the difference is still considerable). Showing Greenland at a slightly greater scale improves the legibility of its coast. Not that tropical areas lack for attention on the Natural Earth projection: with its equatorial aspect and when centered on the Greenwich Meridian, the projection yields Africa-centric maps. The central location and regular outline of that continent invariably attract the reader's eyes.

The Natural Earth projection was designed specifically for making maps centered on the Equator and Greenwich Meridian, 0 latitude, 0 longitude. The distribution of the continents when centered there has a pleasing balance and symmetry-especially Antarctica. (That the Greenwich Meridian is an ideal place to graphically divide the world is entirely good luck). The 180-degree meridians bisect the Ross Sea, indenting the coast on the left and right map margins to make the Antarctic continent appear less large. The coast also trends in the same direction as the meridians converging toward the South Pole, emphasizing the projection shape. Symbolism is also evident in the shape of Antarctica, which appears as a pair of white-gloved hands holding a precious object-Earth-and directs the reader's eyes north across the Southern Ocean toward warmer regions. The effect is not unlike the skies on Heinrich Berann's alpine panoramas with carefully positioned clouds that draw the reader's eyes toward landscape features of interest (Patterson, 2000). Moving the Natural Earth projection center point only 20 degrees to the east or west ruins this effect.

It is the authors' hope that Flex Projector will democratize the creation of world map projections and encourage users to develop innovative and useful new map projections, as Arthur Robinson did nearly 50 years ago. For the first time ever, a user-friendly tool is available to do this.

While designing the A4 and the Natural Earth projection with Flex Projector, we identified possible interface enhancements that could further ease the making of new projections. Placing the sliders directly on the graticule would eliminate the need for a separate panel alongside the map. Or the graticule could itself be adjustable- the user could design a projection by manipulating the graticule by dragging its nodes with the mouse. Also, enhanced interpolation methods could support irregularly spaced control points. The user could then freely add points to the bound- 
ing meridian where needed, similar to how Bézier curves behave in vector drawing programs.

Exchanging projection files created in Flex Projector with other mapping applications would be useful. Since the software is open-source, developers can extract portions of the code and extend their applications to read and interpret descriptions of projections designed with Flex Projector. By doing this, other mapping applications need not provide tools for the design of new projections.

Having an application like Flex Projector freely available to everyone is bound to create some problems. For instance, with the proliferation of new projections it is a certainty that a few will have shoddy designs, including peculiar shapes and large amounts of distortion that the reader is not aware of. The need to document new map projections is another concern. Without the text file that describes a projection created in Flex Projector, the projection is not reproducible nor will it register with other projections. Then there is the challenge of what to call a new projection. Instead of the convention of naming a projection after oneself, some users will opt for more descriptive and eclectic names. The authors of this paper broke with convention when naming the A4 and Natural Earth projections.

The problems mentioned above, however, are minor when weighed against the benefit that Flex Projector brings to cartography: a simple means to create new map projections. Over the last two decades sophisticated technology has made other subfields of cartography accessible to non-specialists, and the profession has adapted as a result. Flex Projector continues this trend.

The authors wish to thank the anonymous reviewers for their valuable comments, Richard Furno, (Azimuth Inc.) for generously sharing his excellent vector data, as well as Daniel Strebe (Mapthematics LTD) and Hans Walser (University of Basel) for their advice and comments. Thanks go also to Gerald I. Evenden for making the Proj4 library publically available and to Jerry Huxtable for porting this library to Java. We also acknowledge the Swiss National Science Foundation for partially financing this project.

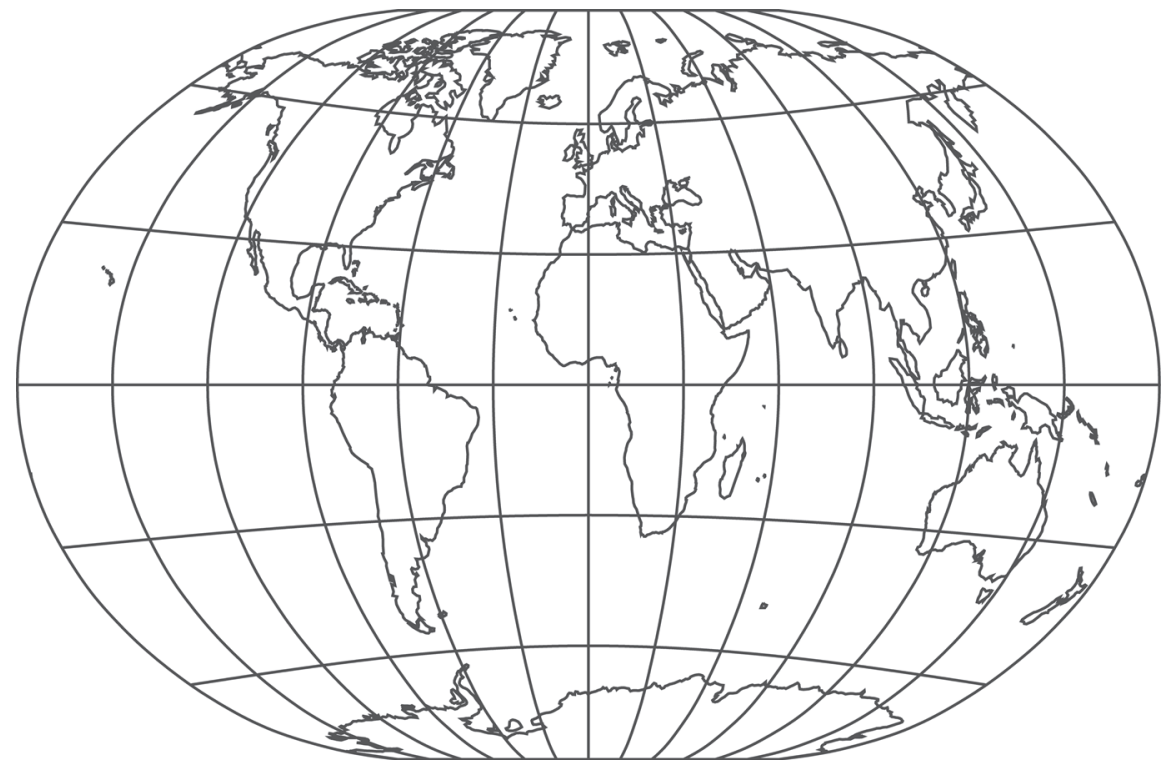

Figure 7. The A4 projection.
ACKNOWLEDGMENTS

\author{
See Appendix A for the \\ Parameters of the A4 Projection
}




\section{APPENDIX A: PARAMETERS OF THE A4 PROJECTION}

The following table lists the parameters for the A4 projection for Flex Projector 1.0. Note: the A4 projection uses a linear distribution of meridians. These values equal 0 and are not listed here.

\begin{tabular}{|c|c|c|c|}
\hline Latitude & $\begin{array}{l}\text { Length of } \\
\text { Parallels }\end{array}$ & $\begin{array}{l}\text { Distance of } \\
\text { Parallels } \\
\text { from } \\
\text { Equator }\end{array}$ & $\begin{array}{l}\text { Bending of } \\
\text { Parallels } \\
\text { (Cosine) }\end{array}$ \\
\hline 0 & 1 & 0 & -0.2218 \\
\hline 5 & 0.998 & 0.075 & -0.2214 \\
\hline 10 & 0.991 & 0.1496 & -0.2198 \\
\hline 15 & 0.98 & 0.2235 & -0.2166 \\
\hline 20 & 0.965 & 0.2955 & -0.2123 \\
\hline 25 & 0.946 & 0.366 & -0.2068 \\
\hline 30 & 0.922 & 0.435 & -0.2 \\
\hline 35 & 0.895 & 0.502 & -0.1919 \\
\hline 40 & 0.864 & 0.567 & -0.1824 \\
\hline 45 & 0.828 & 0.629 & -0.1716 \\
\hline 50 & 0.789 & 0.6885 & -0.1593 \\
\hline 55 & 0.745 & 0.746 & -0.1455 \\
\hline 60 & 0.697 & 0.801 & -0.1301 \\
\hline 65 & 0.647 & 0.85 & -0.113 \\
\hline 70 & 0.596 & 0.893 & -0.0943 \\
\hline 75 & 0.54 & 0.93 & -0.0737 \\
\hline 80 & 0.479 & 0.959 & -0.0512 \\
\hline 85 & 0.415 & 0.982 & -0.0267 \\
\hline 90 & 0.333 & 1 & 0 \\
\hline \multicolumn{3}{|c|}{ Height / width } & 0.655 \\
\hline \multicolumn{3}{|l|}{ Scale } & 0.7785 \\
\hline \multicolumn{3}{|c|}{ Direction of meridians at poles } & $62^{\circ}$ \\
\hline
\end{tabular}




\section{APPENDIX B: PARAMETERS OF THE NATURAL EARTH PROJECTION}

The following table lists the parameters for the Natural Earth projection for Flex Projector 1.0. Note: the Natural Earth projection does not bend parallels and uses a linear distribution of meridians. These values equal 0 and are not listed here.

\begin{tabular}{|c|c|c|}
\hline Latitude & $\begin{array}{l}\text { Length of } \\
\text { Parallels }\end{array}$ & $\begin{array}{l}\text { Distance of } \\
\text { Parallels } \\
\text { from } \\
\text { Equator }\end{array}$ \\
\hline 0 & 1 & 0 \\
\hline 5 & 0.9988 & 0.062 \\
\hline 10 & 0.9953 & 0.124 \\
\hline 15 & 0.9894 & 0.186 \\
\hline 20 & 0.9811 & 0.248 \\
\hline 25 & 0.9703 & 0.31 \\
\hline 30 & 0.957 & 0.372 \\
\hline 35 & 0.9409 & 0.434 \\
\hline 40 & 0.9222 & 0.4958 \\
\hline 45 & 0.9006 & 0.5571 \\
\hline 50 & 0.8763 & 0.6176 \\
\hline 55 & 0.8492 & 0.6769 \\
\hline 60 & 0.8196 & 0.7346 \\
\hline 65 & 0.7874 & 0.7903 \\
\hline 70 & 0.7525 & 0.8435 \\
\hline 75 & 0.716 & 0.8936 \\
\hline 80 & 0.6754 & 0.9394 \\
\hline 85 & 0.627 & 0.9761 \\
\hline 90 & 0.563 & 1 \\
\hline \multicolumn{2}{|c|}{ Height / width } & 0.52 \\
\hline \multicolumn{2}{|l|}{ Scale } & 0.8707 \\
\hline \multicolumn{2}{|c|}{ Direction of meridians at poles } & $60^{\circ}$ \\
\hline
\end{tabular}




\section{APPENDIX C: DISTORTION TABLES FOR THE A4 AND THE NATURAL EARTH PROJECTIONS}

Below are three tables comparing the A4 and the Natural Earth projections to other widely used projections. Note: lower distortion values are better. For details on the computation of these distortion values, see Canters and Decleir (1989).

Weighted mean error for overall scale distortion

\begin{tabular}{|l|l|}
\hline Kavraiskiy VII & 0.23 \\
\hline Natural Earth & 0.25 \\
\hline Winkel Tripel & 0.26 \\
\hline Robinson & 0.27 \\
\hline Plate Carrée & 0.29 \\
\hline A4 & 0.30 \\
\hline Eckert IV & 0.36 \\
\hline Miller Cylindrical & 0.39 \\
\hline Mollweide & 0.39 \\
\hline
\end{tabular}

Weighted mean error for areal distortion

\begin{tabular}{|l|l|}
\hline Eckert IV & 0 \\
\hline Mollweide & 0 \\
\hline A4 & 0.15 \\
\hline Winkel Tripel & 0.18 \\
\hline Robinson & 0.19 \\
\hline Natural Earth & 0.19 \\
\hline Kavraiskiy VII & 0.28 \\
\hline Plate Carrée & 0.57 \\
\hline Miller Cylindrical & 1.30 \\
\hline
\end{tabular}

Mean angular deformation index

\begin{tabular}{|l|l|}
\hline Miller Cylindrical & 7.63 \\
\hline Plate Carrée & 16.84 \\
\hline Kavraiskiy VII & 19.15 \\
\hline Natural Earth & 20.56 \\
\hline Robinson & 21.26 \\
\hline Winkel Tripel & 23.28 \\
\hline A4 & 27.38 \\
\hline Eckert IV & 28.73 \\
\hline Mollweide & 32.28 \\
\hline
\end{tabular}


Bosacki, C., 2007. Comments offered during the Gratuitous Mapping panel at NACIS 2007, St. Louis, MO.

Čapek, R., 2001. Which is the best projection for the world map? Proceedings of the $20^{\text {th }}$ International Cartographic Conference ICC 2001 Beijing China, 5:3084-3093.

Canters, F., 2002. Small-scale map projection design, Taylor \& Francis, London.

Evenden, I. E., 2005. libproj4: A comprehensive library of cartographic projection functions (preliminary draft) (online), http:/ / members.verizon. net/ vze2hc4d/proj4/manual.pdf (Accessed 15 February 2008).

Garver, J. B., 1988. New perspective on the world, National Geographic, 174:6:910-913.

Huxtable, J., 2007. JH Labs_Java Map Projection Library (online), www.jhlabs.com/java/maps/proj/index.html (Accessed 15 February 2008).

Jenny, B. and Patterson, T., 2007. Flex Projector (online), www.flexprojector. com (Accessed 15 December 2007).

Robinson, A., 1974. A new map projection: Its development and characteristics, in Kirschbaum, G.M. and Meine, K.H. (Eds.) International Yearbook of Cartography, Kirschbaum, Bonn-Bad Godesberg, Germany, 145-155.

Patterson, T., 2000. A View from on High: Heinrich Berann's Panoramas and Landscape Visualization Techniques for the U.S. National Park Service. Cartographic Perspectives. 36:38-65.

Patterson, T., 2007. Natural Earth II (online), www.shadedrelief.com/ natural2/ (Accessed 17 November 2007).

Strebe, D., 1999. Geocart Owner's Manual Version 2.6. http:/ / www.macmaps.com/pdflib/manual.pdf (Accessed 15 December 2007).

Snyder, J. P., 1993. Flattening the earth. Two thousand years of map projections, University of Chicago Press, Chicago \& London. 